\title{
Modelos de Cuidados en Escuelas de Formación de Enfermería en la Región Caribe
}

\section{Nursing Care Models in Training Schools from Caribbean Region}

\author{
Gloria Lastre Amell ${ }^{1}$ \\ Universidad Simón Bolívar \\ Gladys Gaviria García ${ }^{2}$ \\ Universidad Simón Bolívar
}

\section{RESUMEN}

Introducción: En los últimos 50 años, los modelos de cuidados en enfermería desde las escuelas de formación se han convertido en tema importante, incitando al desarrollo notable y a la expansión de la formación y la descripción de la disciplina. Objetivo: Caracterizar los modelos de cuidados en enfermería a nivel epistemológico, en búsqueda de una identidad regional acorde con los paradigmas en enfermería. Materiales y métodos: Estudio descriptivo en 158 profesionales de los programas de enfermería de las diferentes universidades de la región Caribe. El instrumento de evaluación fue diseñado por las autoras con base en los postulados de María Mercedes Duran de Villalobos (9). Para el procesamiento de datos, se utilizó el programa SPSS, versión 17. Resultados: El $40 \%$ de los docentes de enfermería reveló que la institución universitaria donde laboran no tiene un modelo y teoría para la enseñanza, pero el 58\% manifestó que sí. De éstos, el 37\% identificó la teoría del Autocuidado; el 11\%, la del Entorno; el 8\%, la Transcultural; y el 2\%, la de Seres Unitarios. Además, el $89 \%$ de los profesionales identificó la relación de los modelos y teorías en la enseñanza y práctica de enfermería. Conclusión: El estudio evidenció que los profesionales identificaron la relación en la enseñanza y la práctica de enfermería y la definición de los modelos y teorías. Los modelos están establecidos en el micro-currículo de las universidades, lo cual es de gran importancia en la formación de los profesionales desde el contexto disciplinar.

Palabras Clave: cuidado, enfermería, modelos, teorías (Fuente: DeCS).

\begin{abstract}
Introduction: In the last 50 years, nursing care models in training schools have become an important issue, prompting remarkable development and expansion of the training and description of the discipline. Objective: To epistemologically characterize the nursing care models, in search of a regional identity in accordance with nursing paradigms. Materials and methods: Descriptive study on 158 professionals from different universities of the Caribbean region nursing programs. The assessment tool was designed by the authors based on the postulates of Maria Mercedes Villalobos. For data processing, the program SPSS version 17 was used. Results: $40 \%$ of the nursing professors claimed that the university where they worked did not have a model and theory for teaching, but $58 \%$ stated the opposite. These identified the following theories: of Self-Care (37\%); the Environment (11\%); Cross-cultural theory (8\%); and Unitary Beings (2\%). In addition, $89 \%$ of the professionals identified the relationship between models and theories and the teaching and practice of nursing. Conclusion: The study proved that the professionals identified the relationship between the teaching and practice of nursing and the definition of the models and theories. The models are established in the micro-curriculum of universities, which is of great importance to the training of nursing professionals within a disciplinary context.
\end{abstract}

Keywords: care, nursing, models, theories (Source: MeSH, NLM).

Enfermera. MSc en Salud Pública. Correo electrónico: glastre@ unisimonbolivar.edu.co

Enfermera. Mg en Educación. Correo electrónico: ggaviria1@unisimonbolivar.edu.co 


\section{Introducción}

La El objeto de estudio de la enfermería es el cuidado. Éste direcciona la práctica y la proyección profesional. Cuidar es un verbo que denota acción, de la que a la vez se deriva un resultado o intervenciones, una ciencia, y un arte o un hecho humano (1). El cuidado en enfermería se fundamenta en modelos y teorías, que no solo son esenciales para su existencia como disciplina académica, sino para la práctica profesional.

Por otra parte, como disciplina, la enfermería se sustenta en un conocimiento teórico que fundamenta todas sus áreas (2); por ello, se soporta en el uso de modelos conceptuales o teorías en la atención, lo que garantiza que las enfermeras y enfermeros compartan igual concepción del meta-paradigma en la orientación de los cuidados (3).

Los modelos permiten construir conocimiento para acceder a un cuidado diferenciado y específico. También apoyan la organización y la ejecución de las actividades planeadas para brindar atención al individuo y/o colectivos. Al mismo tiempo, impulsan el conocimiento y desarrollo del enfermero, por cuanto involucran un conjunto de saberes que optimizan la praxis de enfermería (4).

Los modelos conceptuales explican los constructos de la enfermería y orientan la formación y la investigación del cuidado; igualmente, estos modelos surgen a partir de los postulados de la biología, la psicología, la sociología y la antropología, que explican la estructura del cuidado de enfermería y guían los procesos de formación, investigación y práctica de la profesión $(4,5)$.

Tomando en cuenta su mayor o menor nivel de abstracción, los modelos y teorías se dividen en meta paradigmas, filosofías, modelos conceptuales, grandes teorías y teorías de nivel medio (2). Desde esta óptica, la aplicación de los modelos de enfermería en la enseñanza y durante la práctica logra su éxito a través del acervo, la reflexión y, finalmente, la aplicación de los mismos.

Kuerten (6) expresa que la evolución de la enfermería y su presente implica, más que vocación y buena voluntad, conocimiento. De ahí el interés de hacer una caracterización del cuidado de enfermería a nivel epistemológico. Es de gran importancia, pues, que los profesionales de la enfermería tengan claros los principios científicos, filosóficos y epistemológicos que orientan la práctica, de modo que actúen en concordancia con el ejercicio profesional.

El modelo conceptual que se adopta en un plan curricular proporciona la guía para el diseño de los planes de estudios y de las estrategias de enseñanzaaprendizaje en la educación en enfermería; en el caso particular de la aplicación de un modelo en la Facultad de Enfermería de la Universidad de la Sabana, se realizó un estudio exhaustivo de los modelos que habían tenido un mayor nivel de desarrollo y cuya filosofía estuviera acorde con los principios expresados en el proyecto educativo de la universidad y en el programa (7).

De manera similar, el presente estudio es importante porque aporta a la consolidación de los modelos y teorías de enfermería en escuelas de formación de la región Caribe, los cuales ofrecen elementos conceptuales prácticos que ayudan a estudiantes de pregrado y postgrado, así como a profesionales de enfermería, en su aprendizaje y ejercicio profesional. Por ello, el objetivo de este artículo consistió en caracterizar los modelos de cuidado en enfermería a nivel epistemológico, en búsqueda de una identidad regional acorde con los paradigmas de la disciplina.

\section{Materiales y Métodos}

El nuestro es un estudio descriptivo, realizado en 158 profesionales de las áreas, que comprenden directivos, administrativos y docentes de los programas de enfermería de las diferentes universidades de la región Caribe. Se realizó un muestreo por conveniencia para seleccionar a los sujetos, quienes aceptaron participar en el estudio de manera voluntaria.

Los sujetos de estudio diligenciaron una encuesta estructurada e integrada por nueve preguntas con opciones de selección múltiple. El instrumento de evaluación fue diseñado por las autoras con base en los postulados de María Mercedes Duran de Villalobos (8), y comprende subdimensiones, identificando los conceptos de definición de la profesión como ciencia. Además, se resalto el rol de modelos y teorías en enseñanza y la práctica de enfermería, así como la identificación de modelos y teorías que aplica cada institución de la región Caribe, conceptos meta paradigmáticos y la estructura interna que integran los 
modelos y teorías de enfermería. Para el procesamiento de datos, se utilizó el programa SPSS, versión 17.

\section{Resultados}

El rango de edades del $30 \%$ de la población, que comprendió al mayor porcentaje, se fijó en 50 años y más. En cuanto al género, el $98 \%$ correspondió al sexo femenino.

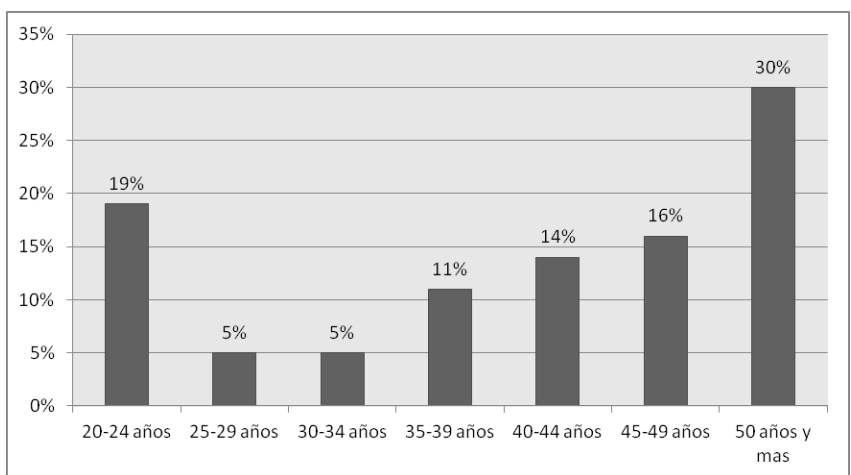

Figura 1. Rango de edad de la población en estudio

El 58\% de los docentes de enfermería indicó que la institución universitaria donde labora sí cuenta con un modelo y teoría para la enseñanza

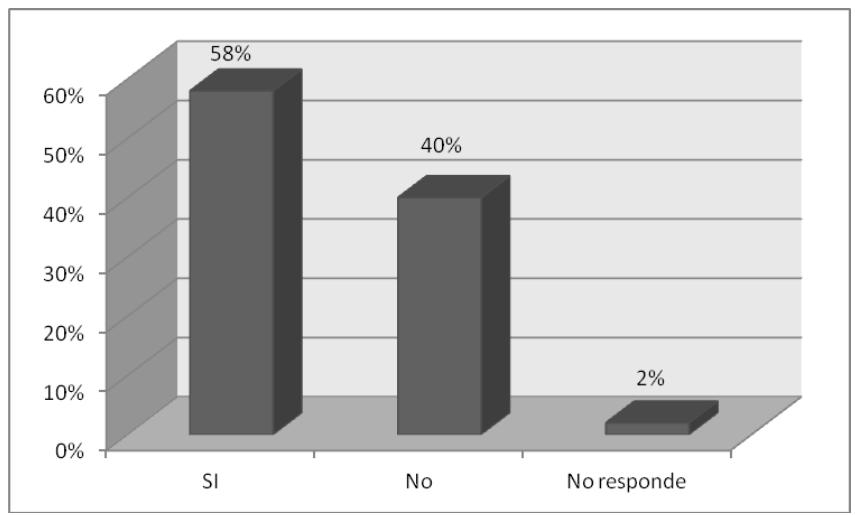

Figura 2. Existencia de un modelo y teoría que guía la enseñanza
De otro lado, el $89 \%$ de los profesionales docentes de enfermería identificó acertadamente el papel de los modelos y teorías en la enseñanza y en la práctica de la profesión.

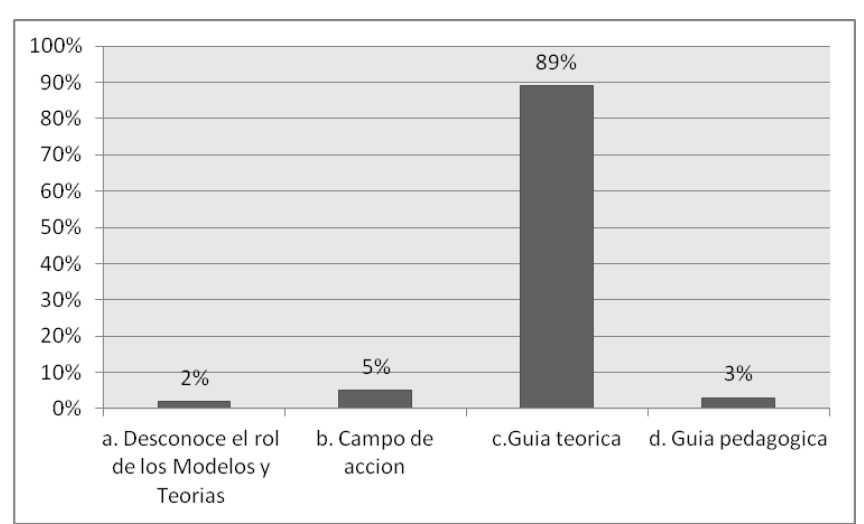

Figura 3. Rol de los modelos y teorías en la enseñanza y práctica de Enfermería

En cuanto a los modelos y teorías identificados por los docentes de enfermería, un $37 \%$ señaló la teoría del Autocuidado y sólo el $2 \%$ escogió la teoría de los Seres Unitarios.

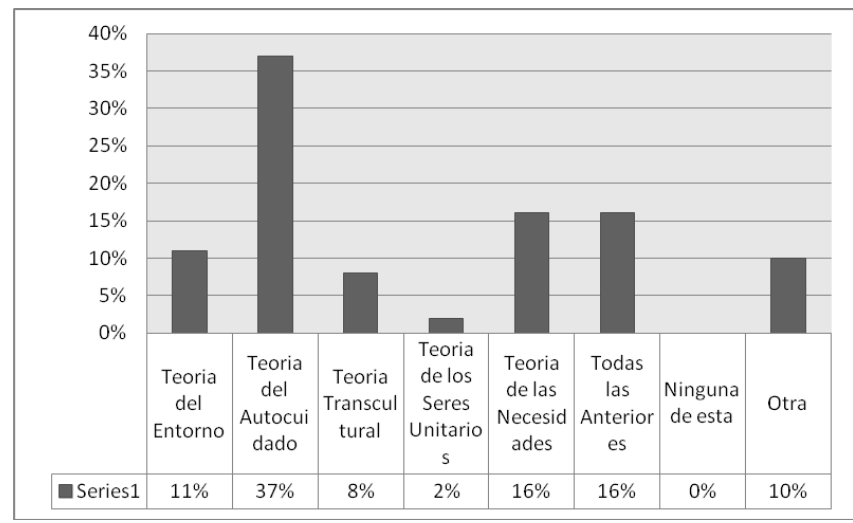

Figura 4. Identificación de los Modelos y Teorías

El $60 \%$ de los docentes de enfermería manifestó que le gustaría que existiera un modelo y teoría que identifique el programa de enfermería en el que labora. 


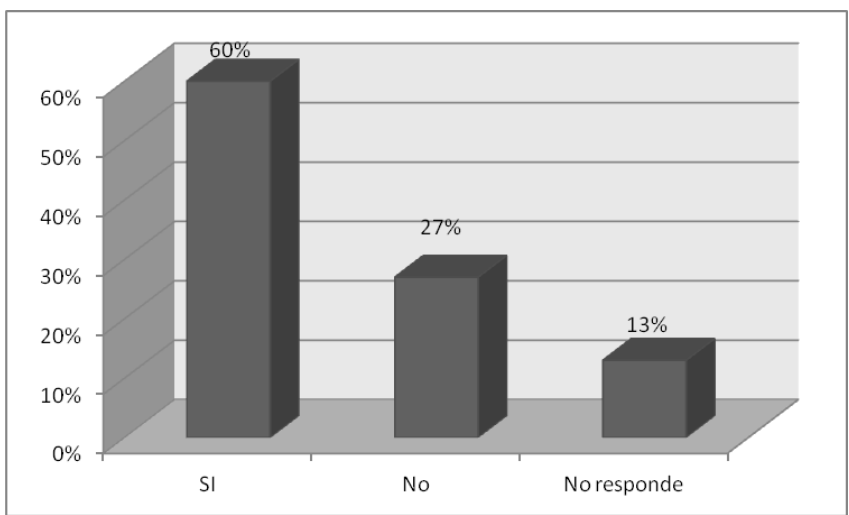

Figura 5. Preferencia de los docentes por la existencia de un modelo y teoría específico que identifique el programa de formación

\section{Discusión}

Los resultados muestran un mayor porcentaje de profesionales de enfermería mayores de 50 años y de género femenino, cuestión que confirma el estudio de Pimentel (9), en el cual se reconoce que durante años la práctica de los cuidadores se ha asociado a los numerosos papeles atribuidos a la mujer, que es un fruto de la división social del trabajo mismo.

La presente investigación confirmó la necesidad de desarrollar de manera permanente investigaciones inherentes a este tema, que permitan fortalecer métodos de enseñanza-aprendizaje que contribuyan al progreso de la disciplina y profesión, aportando de esta manera a una mejor prestación de los servicios de enfermería en los diferentes campos de acción (10).

Este estudio identificó que los modelos y teorías guían la enseñanza y la práctica de la profesión; lo cual coincide con lo expuesto por Amaro (11), las enfermeras $y$ enfermeros han modificado sus desempeños, desde la segunda mitad del siglo XX, debido al incremento de sus conocimientos teóricos. La autora considera que los conceptos teóricos, han logrado que los profesionales de enfermería brinden el cuidado, a través de técnicas y herramientas que le permiten diagnosticar, identificar situaciones problemas del individuo, familia y colectivos (11).

Lo anterior también se puede contrastar con lo anotado en el artículo de Seguel (12), el cual señala que la enfermería ha sido definida como una profesión, disciplina y ciencia, con sus propias corrientes filosóficas que incluyen conceptos metaparadigmáticos como: persona, entorno, salud y cuidado; este autor resalta el paradigma de categorización, el cual tiene como base de su funcionamiento la relación causal lineal, estableciendo que las características de los fenómenos se definen a partir de una metodología deductiva y lógica.

Por otro lado, la mitad de los docentes de enfermería indicó que en la institución universitaria donde labora sí tienen un modelo y teoría para la enseñanza, dato que se conjuga con el estudio de Moreno (13), porque de acuerdo con los avances de las ciencias, los teóricos de la disciplina son los que definen los modelos y teorías propios de enfermería, toda vez que permiten explicar la atención de las necesidades de cuidado de las personas, de las familias y de grupos de la comunidad.

En relación con la variable que indagaba si los docentes de enfermería identifican el papel de los modelos y teorías en la enseñanza y en la práctica, un alto porcentaje de los profesionales así lo hizo. Este resultado concuerda con lo anotado por otros autores (14) estimando que la enfermería es una ciencia aplicada, es decir, su práctica implica un conocimiento teórico y principios filosóficos y éticos, logrando ser una profesión autónoma que participa de manera activa en la toma de decisiones de las políticas públicas de salud.

Asimismo, los profesionales identificaron, en primer lugar, la teoría del autocuidado en la institución donde trabaja. Esta teoría se incluye dentro de los modelos conceptuales de enfermería, y han sido llamadas grandes teóricas o pioneras en este campo porque explican toda la enfermería e incluyen aspectos relativos a los seres humanos, su entorno y su salud (10).

Un porcentaje considerable expresó que le gustaría que existiera un modelo y teoría que identifique el programa de enfermería en el que labora, respuesta que se inscribe en la línea de lo asentado en el artículo de Santos (15); dicho artículo plantea que los modelos conceptuales explican los fenómenos y definen los límites entre las profesiones; este autor define como complejo el cuerpo filosófico de enfermería, ya que existe un número de modelos conceptuales y teorías, donde algunas son de difícil comprensión; y algunos modelos están alejados de la labor diaria de estos profesionales, quienes en muchas ocasiones deben adaptar los recursos disponibles a sus necesidades (15). 
Es ineludible, entonces, que las diferentes escuelas de formación establezcan una identidad en la formación de sus profesionales de enfermería, una identidad que los caracterice desde su modelo y teoría. Pero, precisamente, los resultados de este estudio evidenciaron que los programas de enfermería de la región Caribe tienen debilidades al respecto, aun cuando es claro que en la formación del profesional son importantes las estructuras de conceptos fundamentados en contextos epistemológicos y filosóficos.

A pesar de todo, llama la atención que un porcentaje considerable de docentes universitarios de la región Caribe respondió de manera negativa a la posibilidad de que exista un modelo y teoría que identifique el programa de enfermería en el cual labora, aspecto neurálgico por cuanto las modelos y teorías cumplen un rol muy significativo para los profesionales tanto en su formación como en su práctica.

Por último, cabe destacar que la práctica basada en la ciencia soporta a la enfermería como una disciplina profesional, y proporcionan una base para las que las acciones de enfermería no sean estereotipadas y rutinarias (16).

Teniendo en cuenta los conceptos de auto cuidado expresados por Dorotea Orem, Pereda (17) manifiesta que la funcionalidad vital de la persona mediante el cuidado de sí misma es la noción clave de esta teoría y por sus características propias puede utilizarse en personas con y sin problemas de salud por cuanto puede facilitar las actividades de promoción de la salud.

En conclusión, las tendencias o modelos no se reflejan repentinamente, requieren esfuerzos $y$ reformas curriculares para que desde los programas de formación se fortalezca la disciplina de la enfermería. He aquí la importancia de modelos conceptuales que aporten, a través del conocimiento, herramientas y estrategias para aplicarlas en el ámbito asistencial, ambulatorio y educativo, sobre todo en este último, para hacer frente al reto de la disciplina ante los diferentes cambios, ya sean económicos, políticos o culturales.

\section{Referencias}

1. Duran MM. El arte y la ciencia del cuidado. Teorías de rango medio importancia y utilidad para la práctica de enfermería. Segunda edición. Bogotá: Universidad Nacional de Colombia; 2010.

2. Varez PS, López PM, Santos RS, Abril SD. Perspectiva sobre modelos y teorías de enfermería en el ámbito de nefrología. Rev Soc Esp Enferm Nefrol. 2008; 11(3): 178-183.

3. Marriner TA, Raile M. Modelos y teorías en enfermería. $5^{\text {a }}$ ed. Madrid: Mosby; 2005.

4. Marriner TA, Raile M. Modelos y teorías en enfermería. $7^{\mathrm{a}}$ ed. Madrid: Elsevier Mosby; 2011.

5. Grupo de Cuidado Facultad de Enfermería. Dimensiones del Cuidado. Bogotá: Universidad Nacional de Colombia, Unibiblios; 2007.

6. Kuerten RP, Lenise PM. Modelo de Cuidado ¿Qué es y como elaborarlo? Index Enferm. 2008; 17 (2): 128-132.

7. Moreno FM. Importancia de los modelos conceptuales: experiencia de la Facultad de Enfermería Universidad de la Sabana. Aguichan. 2005; 5 (1): 44-45.

8. Duran MM. La teoría, soporte de la ciencia y práctica de enfermería: tendencias. Av.enferm. 2012; 30(1): 9-12.

9. Pimentel MH, Pereira FA, Da Mata MA. La construcción de la identidad social y profesional de una profesión femenina: Enfermería. Revista Sociology. 2011; 7: 1-23.

10. Rodríguez MR. Modelos de enfermería integrados al cuidado del paciente nefrológico en el contexto cubano. Rev. Cubana Enfermer. 2012; 28(4): 474484.

11. Amaro CM. Florence Nightingale, la primera gran teórica de enfermería. Rev. Cubana Enfermer. 2004; 20 (3): 0-0.

12. Seguel PF, Valenzuela SS, Sanhueza AO. Corriente epistemológica positivista y su influencia en la generación del conocimiento de enfermería. Aquichan. 2012; 12 (2): 160-168.

13. Moreno FM. Metodologías para la aplicación del conocimiento teórico de enfermería. Aquichan. 2012; 12(2): 95-96.

14. Navarro PY, Castro SM. Modelo de Dorothea Orem aplicado a un grupo comunitario a través del 
proceso de enfermería. Enferm. glob. 2010; 9 (2): $1-14$.

15. Santos RS, López PM, Sánchez RG, Bardallo PL, Fernández DL, Zabalegui YA, et al. Modelos teóricos y estudiantes de enfermería: aspectos motivacionales para su elección durante las prácticas clínicas. Educ. méd. 2011; 14(2): 119127.

16. Reyes LJ, Jara CP, Merino EJ. Adherencia de las enfermeras/os a utilizar un modelo teórico como base de la valoración de enfermería. Cienc. enferm. 2007; 13(1): 45-57.

17. Pereda AM. Explorando la teoría general de enfermería de Orem. Enf Neurol. 2011; 10 (3): 163-167. 